\title{
Bioanalysis
}

\section{Immunoaffinity MS: adding increased value through hybrid methods}

\author{
"The promise of IA-MS is best realized through analytical \\ performance or opportunities that cannot be achieved using its \\ component parts. Hence, it is important to explore novel applications \\ for IA-MS in areas not previously considered or viewed as tenable."
}

First draft submitted: 2 June 2016; Accepted for publication: 13 June 2016;
Published online: 20 July 2016

Keywords: biotherapeutics $\bullet$ hybrid methods $\bullet$ immunoaffinity $\bullet$ immunoprecipitation - LC-MS MALDI • protein biomarkers

It gives me great pleasure to introduce a special focus issue in Bioanalysis devoted to immunoaffinity MS (IA-MS) methods. The combination of IA sample preparation with MS detection is now over two decades old and has evolved considerably since the introduction of the mass spectrometric immunoassay format by Nelson and co-workers in 1995 [1]. As described in this issue, the power inherent in the combination of these two methods is allowing analytical researchers to keep pace with advances in biological sciences and in many cases creating new opportunities for research.

With publications, including white papers chronicling the progress of IA-MS methods, also referred to as hybrid techniques [2-4], it is fair to say that IA-MS is now a fully recognized analytical genre. With that said, in many ways we now stand at a pivotal juncture in the history of IA-MS. Although IA-MS has become increasingly commonplace in pharmaceutical, biotech and academic laboratories, it is far from mature in terms of preferred applications, techniques or best practices. At the same time, IA-MS is also a long way from being universally adopted. While this leads to excitement about the future of IA-MS, it is clear that the next few years will be critical to its development and long-term use.

At the heart of IA-MS is the ability to deliver analytical performance not found with ligand-binding assays (LBA) or MS alone. LBA has inherent limitations in analyte specificity, while MS assays in complex biological matrices face limited sensitivity without the use of IA capture. Although the complementary nature of IA-MS would seem to make it the logical choice for many applications, the merger of these two methods has not always been straightforward and adoption in many cases is challenged by the inherent complexity of the required methodology. So where does IA-MS head from here? I suggest investment along two separate but interdependent paths:

\section{Continue to exploit the inherent value of IA-MS}

The promise of IA-MS is best realized through analytical performance or opportunities that cannot be achieved using its component parts. Hence, it is important to explore novel applications for IA-MS in areas not previously considered or viewed as tenable. A clear example is found in the research article by Leigh Anderson, which exploits the long standing advantage of IA-MS to deliver highly quantitative multiplexed analysis [5]. Using a SISCAPA approach (stable isotope standards and capture by anti-peptide antibodies [6]), Anderson demonstrates the capability for intrasubject longitudinal analysis of a panel of 22 clinically relevant proteins in dried blood spots. Because intrasubject biomarker changes are often more diagnostic than a comparison to population-derived

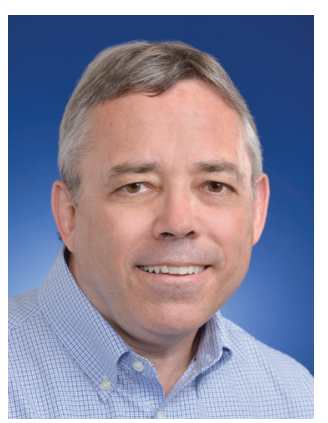

Bradley L Ackermann Investigative Toxicology, Eli Lilly \& Company, Lilly Corporate Center, Drop Code 0720, Indianapolis, IN 46285, USA Tel.: +1 3172764836

brad.ackermann@lilly.com 
values, the power of this technology could lead to a new paradigm for clinical diagnostic testing.

A second illustration appears in a commentary [7] by Hendrik Neubert, who advocates for the use of IA-MS by SISCAPA to quantify targets inaccessible to conventional antiprotein capture. One area highlighted is tissue analysis, including formalin-fixed paraffin embedded samples and the analysis of membrane bound proteins. The utility of this approach was demonstrated in a recent publication by this group involving quantification of human neonatal $\mathrm{Fc}_{\mathrm{c}}$ receptor expression in tissue from a transgenic mouse model [8]. The information derived was used to support physiologically based PK (PBPK) models to predict therapeutic antibody clearance.

The relative simplicity and reliability of IA-MS for multianalyte analysis appears to be a common theme for value generation. Two additional examples appear in this issue from pharmaceutical laboratories. In contrast to the work of Anderson, these examples use antiprotein antibodies to capture multiple proteoforms of a target analyte. Using high resolution-accurate mass (HR-AM) on an orbitrap mass spectrometer Cox and co-workers demonstrate the ability to simultaneously quantify the incretin hormone oxyntomodulin and its primary hydrolysis products in both human and rat plasma [9]. This work is complemented by a PK example by Mehl and colleagues who used IA-MS to simultaneously quantify monoclonal antibody drug candidates in cynomolgus monkey plasma along with specific breakdown products known to affect target binding originating from labile asparagine or aspartate residues [10]. In both of these cases the ability to deliver additional insight about the target molecule with comparatively limited method development and cost speaks to the inherent value of IA-MS.

\section{Promote wider adoption}

The second area of focus needs to be on finding opportunities to expand the uptake of IA-MS. In many ways this objective presents a greater challenge as it involves issues which are both technical and nontechnical in nature. Among technical issues is the need for even greater sensitivity, as exemplified by the frequent use of exaggerated sample sizes (e.g., 0.1-1 ml) for clinical biomarkers. Clearly, further advances in MS ionization and detection will be required. Improvements in sensitivity are vital as they also lead to simpler method development and decreased reliance on techniques such as nano-LC. In this context, the recent trend toward HR-AM is an important area worthy of further exploration. In the method presented by Cox and co-workers [9] ultra-high resolution detection $(70,000$ FWHM) in full scan mode was used to allow fasting levels of oxyntomodulin to be measured in human plasma with narrow bore LC (2.1 mm id). An important feature of this work is that the significant signal dilution introduced by peptide CID was avoided suggesting that the full scan HR-AM has the potential for both improved sensitivity and ease of operation.

IA-MS adoption is also encouraged by developing easier methods for sample preparation and notable examples are presented in this issue. The continual need to procure high quality, affordable reagents for antigen capture was represented by Lassman $e t$ al. who make a case for the use of aptamers as an alternative to antibodies for immunocapture [11]. Using subtilisin/kexin type 9 (PCSK9) as a test molecule, data are presented showing comparable performance with the advantage of lower background. Because aptamers are produced via synthetic means, they offer a distinct advantage over antibodies in terms of time required for production.

The importance of sample preparation is further underscored in a review by Nedlekov and Nelson on MALDI-ToF applications of mass spectrometric immunoassay [12]. Here the authors state that front end sample preparation combined with the complexity of LC-MS has limited the uptake of clinical applications for protein biomarkers. In cases where sufficient sensitivity can be obtained, top-down methods using MALDI have a distinct advantage over ELISA due to the ability to differentiate and detect multiple proteoforms of a given biomarker. The chemokine RANTES was used as a representative example along with retinol binding protein and apolipoprotein C-III.

The path to wider adoption is further abetted by simplification and standardization of common workflows. In an article by Kaur et al. a 'plug and play' method to perform monoclonal antibody (mAb) PK in cynomolgus monkey serum is presented [13]. In addition to demonstrating method validation according to recent recommendations [4], the ability to quickly adapt the method to other mAbs and to other preclinical species was demonstrated.

Perhaps the biggest challenges to adoption are nontechnical in nature. Realistically, IA-MS can only expand by having more trained analysts and, owing to the broad skill-set required, this has already emerged as a significant issue. Other barriers include the cost of instrumentation, which can only be offset by increased productivity and identifying applications that require IA-MS. Another concern is assay transfer and the reliability of CRO partners. At the present time, several CROs are actively becoming engaged in IA-MS methods and we are fortunate to have commentary by Jones and Schultz who share insights gained from their experience in this area [14]. 
Finally, as we head into the future, the best opportunities for IA-MS are likely to emerge from laboratories having a combined experience in both LBA and MS methods. This point was made in an editorial by Binodh DeSilva who suggests that the bioanalysts of the future will need to be equipped with experience in both areas [15]. Indeed, such scientists will help chart the course of IA-MS over the next 20 years.

\section{References}

1 Nelson RW, Krone JW, Bieber AL et al. Mass spectrometric immunoassay. Anal. Chem. 67(7), 1153-1158 (1995).

2 Ackermann B, Neubert $\mathrm{H}$, Hughes $\mathrm{N}$ et al. 2015 White paper on recent issues in bioanalysis: focus on new technologies and biomarkers (Part 2 - hybrid LBA/LCMS and input from regulatory agencies). Bioanalysis $7(23)$, 3019-3034 (2015).

3 Duffield D, Neubert H, Garofolo, et al. 2014 White paper on recent issues in bioanalysis: a full immersion in bioanalysis (Part 2 - hybrid LBA/LCMS, ELN and regulatory agencies' input). Bioanalysis 6(23), 3237-3249 (2014).

4 Jenkins R, Duggan JX, Aubry AF et al. Recommendations for validation of LC-MS/MS bioanalytical methods for protein biotherapeutics. AAPS J. 17(1), 1-16 (2015).

5 Razavi M, Anderson NL, Yip R, Pope ME, Pearson TW. Multiplexed longitudinal measurement of protein biomarkers in DBS using an automated SISCAPA workflow. Bioanalysis 8(15) 1597-1609 (2016).

6 Anderson NL, Anderson NG, Haines LR et al. Mass spectrometric quantitation of peptides and proteins using stable isotope standards and capture by anti-peptide antibodies (SISCAPA). J. Proteome Res. 3(2), 235-244 (2004).

7 Neubert H, Fan Y-Y, Ocaña MF. Quantification of protein biomarkers in tissues: new capabilities with pellet digestion peptide immunoaffinity LC-MS/MS. Bioanalysis 8(15) 1551-1555 (2016).

\section{Financial \& competing interests disclosure}

The author has no relevant affiliations or financial involvement with any organization or entity with a financial interest in or financial conflict with the subject matter or materials discussed in the manuscript. This includes employment, consultancies, honoraria, stock ownership or options, expert testimony, grants or patents received or pending, or royalties.

No writing assistance was utilized in the production of this manuscript.

8 Fan YY, Neubert H. Quantitative analysis of human neonatal $\mathrm{Fc}$ receptor $(\mathrm{FcRn})$ tissue expression in transgenic mice by online peptide immuno-affinity LC-HRMS. Anal. Chem. 88(8), 4239-4247 (2016).

9 Cox JM, Berna MJ, Jin Z et al. Characterization and quantification of oxyntomodulin in human and rat plasma using high resolution accurate mass LC-MS. Bioanalysis 8(15), 1579-1595 (2016).

10 Mehl JT, Sleczka BG, Ciccimaro EF et al. Quantification of in vivo site-specific Asp isomerization and Asn deamidation of $\mathrm{mAbs}$ in animal serum using IP-LC-MS Bioanalysis 8(15), 1611-1622 (2016).

11 Gupta V, Lassman ME, McAvoy T, Lee AYH, Chappell DL, Laterza OF. An evaluation of an aptamer for use as an affinity reagent with MS: PCSK9 as an example protein. Bioanalysis 8(15), 1557-1564 (2016).

12 Trenchevska O, Nelson RW, Nedelkov D. Mass spectrometric immunoassays for discovery, screening and quantification of clinically relevant proteoforms. Bioanalysis 8(15), 1623-1633 (2016).

13 Kaur S, Liu L, Cortes DF et al. Validation of a biotherapeutic immunoaffinity-LC-MS/MS assay in monkey serum: 'plug-and-play' across seven molecules. Bioanalysis 8(15), 1565-1577 (2016).

14 Jones BR, Schultz GA. Adaptation of hybrid immunoaffinity LC-MS methods for protein bioanalysis in a Contract Research Organization. Bioanalysis 8(15), 1545-1549 (2016).

15 DeSilva B. Immunoaffinity-coupled MS: best of both technologies. Bioanalysis 8(15), 1543-1544 (2016). 